\section{Mycetoma in Timor-Leste and first report of nocardiosis}

\author{
Nicola Townell, ${ }^{1}$ Thomas Locke, ${ }^{2}$ \\ Margaret Gibbons,3 Dan Murphy, ${ }^{3}$ \\ Joshua Francis, ${ }^{4-6}$ Clare Nourse ${ }^{5,7}$ \\ ${ }^{1}$ Infectious Disease Department, \\ Sunshine Coast University Hospital, \\ Queensland, Australia; ${ }^{2}$ Department of \\ Infection, Sheffield Teaching Hospitals, \\ United Kingdom; ${ }^{3}$ Bairo-Pite Clinic, \\ Bairo-Pite, Timor-Leste; ${ }^{4}$ Infectious \\ Disease Department, Royal Darwin \\ Hospital, Northern Territories, \\ Australia; 5Maluk Timor, Dili, Timor- \\ Leste; ${ }^{6}$ Menzies School of Health \\ Research (Global \& Tropical Health \\ Division), Australia; ${ }^{7}$ Lady Cilento \\ Children's Hospital, Brisbane, Australia
}

\begin{abstract}
Mycetoma is a neglected tropical disease with an unknown global burden. Although considered endemic to South-east Asia, it has not previously been reported from Timor-Lest. We describe two cases in Timor-Leste, highlighting subsistence farming.

We report the first two cases of mycetoma in Timor-Leste in patients attending Bairo Pite Clinic (BPC) in the capital, Dili. $\mathrm{BPC}$ is a non-government organization providing free primary and inpatient care, funded by international donors.
\end{abstract} the challenges surrounding microbiological diagnosis and management shared by many low to middle-income countries. As characteristically described, both patients lived rurally and presented late with marked soft tissue involvement and multiple draining sinuses following a prolonged period of high morbidity. Nocardia brasiliensis, a beadedbranched, modified acid-fast, gram-positive bacilli, was isolated and confirmed by molecular testing in the first case. The causative organism in the second case could not be confirmed due to limited microbiological capabilities. Due to limited local laboratory capabilities, Nocardia spp. infection cannot be routinely confirmed in TimorLeste. However, the microbiology laboratory is essential for the successful diagnosis and management of Mycetoma. In both cases, medical therapy alone resulted in cure and favorable outcomes, although supply of antibiotic remains an ongoing resource issue.

\section{Introduction}

Mycetoma is a neglected tropical disease with an unknown global burden. ${ }^{1}$ Although considered endemic to South-east Asia, it has not previously been reported from TimorLeste. $^{2}$

Mycetoma is a chronic granulomatous destructive soft tissue infection caused by either aerobic actinomycetes or fungi. ${ }^{3}$ Two- thirds are bacterial, with over half caused by Nocardia spp (majority due to Nocardia brasiliensis). ${ }^{4}$ Mycetoma characteristically presents with tumor like swelling and multiple sinuses discharging pus and grains. ${ }^{5}$ Without appropriate therapy, contiguous spread of infection occurs leading to bone involvement with significant morbidity for the patient and results in a detrimental economic impact on the patient's community. ${ }^{3}$ The burden of disease is greatest in rural persons of lower socio-economic status living in the tropical and subtropical regions of the world. ${ }^{3}$

Timor-Leste is a small country in South East Asia with a population of 1.2 million people and an approximate Gross Domestic Product per capita of \$2279 US dollars. Despite significant economic progress, following a long history of devastating conflict and subsequent independence in 2002, nearly $50 \%$ of the population remains in poverty. ${ }^{6}$ The majority of these families reside in rural areas and are primarily engaged in

\section{Case Report \#1}

A 34-year-old previously fit and well progressive left foot swelling and worsening mobility. There was no history of trauma, but he frequently walked barefoot. Examination revealed a swollen left foot with multiple discharging sinuses (Figure 1). Plain radiographs showed no evidence of bony involvement. Empirical trimethoprim-sulfamethoxazole was commenced for suspected nocardiosis. Fresh tissue was brought (by a volunteer) to a laboratory in Australia for histology and culture. Histology examination revealed a mixed inflammatory response with evidence of Splendore-Hoeppli phenomenon. Microorganisms were not visualised on special stains (Gram, Ziehl-Neelsen, Wade Fite, Periodic Acid-Schiff and Grocott).

Bacterial microscopy revealed occative bacilli. Initial cultures grew mixed enteric flora including Pseudomonas aeruginosa and Candida parapsilosis complex. After 5 days, typical white dry pitted colonies were isolated. Gram stain of these colonies revealed a gram-positive, branching filamentous bacilli that were acid-fast stain negative and modified acid-fast stain positive. Subsequent 16 s polymerase chain farmer presented with a two-year history of sional leucocytes and numerous gram-nega-
Correspondence: Nicola Townell, Infectious Disease Department, Sunshine Coast University Hospital, Queensland, Australia. Tel.: +61407485716

E-mail: nikki.microsi@gmail.com

Key words: nocardia; mycetoma; TimorLeste.

Contributions: all authors provided information in respect to the case details. NT drafted the first version of the manuscript. All authors reviewed and approved the manuscript.

Conflict of interest: the authors declare no potential conflict of interest.

Funding: none.

Received for publication: 29 July 2018.

Revision received: 10 October 2018.

Accepted for publication: 19 October 2018.

This work is licensed under a Creative Commons Attribution-NonCommercial 4.0 International License (CC BY-NC 4.0).

CCopyright N. Townell, et al., 2018

Licensee PAGEPress, Italy

Infectious Disease Reports 2018; 10:7804

doi:10.4081/idr.2018.7804

reaction sequencing was performed on the isolate which confirmed Nocardia brasiliensis. The isolate tested in vitro susceptible to trimethoprim-sulfamethoxazole (MIC 0.25/4.8), tobramycin (MIC $\leq 1$ ), amikacin ( $\mathrm{MIC} \leq 1)$ and linezolid (MIC 2), intermediate to amoxicillin-clavulanic acid (MIC 16:8) and minocycline (MIC 4), and resistant to ciprofloxacin ( $\mathrm{MIC}>4$ ), clarithromycin (MIC >16), ceftriaxone (MIC $>64$ ) and imipenem (MIC >64) (Sensititre broth microdilution, CLSI M24-A2). Mycobacterial and fungal cultures were negative. After 2 months of first line treatment targeting Nocardia, the lesions had healed and the patient was able to return to work.

\section{Case Report \#2}

A 45-year-old rural woman presented with a 7 year history of severe pain in her left leg and multiple sinuses draining copious pus (Figure 1). The lesion initially appeared on her foot and over time had slowly progressed caudally to include most of her leg. Due to pain, she was unable to weight bear and she described a very poor quality of life. Surgery had been performed twice without resolution of her symptoms. 
There was no significant past medical history or evidence of immune deficiency (Human Immunodeficiency Virus negative, no diabetes or malignancy). Plain radiographs confirmed osteomyelitis. Due to the high incidence of tuberculosis in TimorLeste (498 cases per 100,000 population), a presumptive diagnosis of tuberculosis osteomyelitis was made and she was commenced on standard quadruple TB therapy. ${ }^{7}$ She experienced ongoing intermittent fevers with increased pus draining from the sinuses. A visiting medical scientist detected branching beaded gram-positive bacilli on a Gram stain of the pus (Figure 2). After 5 days of aerobic incubation (at a private laboratory), chalky colonies with an earthy odor were isolated. The laboratory did not have capabilities to further identify the organism. The isolate was suspected to be Nocardia sp. based on the clinical presentation, characteristic initial gram stain and typical phenotypic appearance. Susceptibility testing was not performed due to limited microbiological capabilities. Initially the patient failed to respond to oral trime thoprim-sulfamethoxazole $(7.5 \mathrm{mg} / \mathrm{kg} /$ day trimethoprim) alone. Subsequently intravenous ceftriaxone (1g daily) was added to therapy with rapid clinical improvement. Ceftriaxone was administered for three months (courtesy of an international donor). She subsequently completed a further 9 months of oral trimethoprim-sulfamethoxazole therapy. On discontinuation of therapy, her lesions had completely healed and she could stand and walk without assistance. Two years after completion of therapy, she was well with no evidence of relapsed infection.

\section{Discussion}

Although Mycetoma is considered endemic in South East Asia, these are the first two reported cases from Timor-Leste. ${ }^{2}$ Clinically the condition is underappreciated and the global burden remains unknown ${ }^{4}$ In recognition of its global importance the World Health Organization (WHO) added Mycetoma to its list of neglected tropical diseases in 2016.

Case 1 was typical of the chronic clinical syndrome known as Madura foot. Nocardia is considered to be a common causative pathogen of this clinical syndrome. ${ }^{5}$ Nocardia spp. when isolated is generally considered a pathogen. ${ }^{8}$ Nocardia brasiliensis was confirmed by isolation and subsequent molecular identification. Molecular testing is useful for confirmation of identification and is considered necessary for accurate species identification. In this case, $16 \mathrm{~s}$ sequencing was performed due to local availability although sequencing of other housekeeping targets such as sec1, gyrB and hsp65 can also be used. ${ }^{9}$ The mixed enteric flora that was isolated is representative of colonization of open wounds. The patient responded to treatment that did not include activity against the other two pathogens, Pseudomonas aeruginosa or Candida parapsilosis, that were isolated. The patient symptoms improved rapidly with complete resolution of infection following the introduction of co-trimoxazole, first line therapy for treatment of Nocardia infection. The clinical presentation, positive culture result, confirmation of pathogen identification by molecular techniques and response to therapy all support the diagnosis of Nocardia infection. This is the first case of microbiologically confirmed Nocardia mycetoma in Timor-Leste.

Patient 2 had many of the clinical signs of Nocardia spp. osteomyelitis with marked soft tissue involvement, multiple sinuses with profuse pus, lytic areas in bone with proliferative periosteal reaction that progresses to sclerosis and the absence of sequestra. ${ }^{10}$ The initial gram stain revealed a branching beaded gram positive bacilli, which is characteristic of Nocardia spp. ${ }^{11}$ When Nocardia spp. infection is suspected, it is important that the laboratory extends incubation of the plates, recommended minimum of 2 weeks, to allow this slower growing organism adequate time to culture. ${ }^{9}$ It is also recommended, particularly for non-sterile sites, that selective media e.g. buffered charcoal yeast extract agar, is used to suppress the growth of other organisms that may mask the presence of this important pathogen. ${ }^{12}$ Although the organism isolated in Case 2 could not be definitively identified due to limited laboratory capabilities, both the initial gram stain and the typical appearance on plates are suggestive of Nocardia sp. A positive modified acid-fast stain (and negative acid-fast stain) would provide further support to the identification of Nocardia (excluding myobacteria and actinomycetes). ${ }^{9}$ Many laboratories in the resource limited setting currently do not have molecular capabilities and so would have to make a presumptive diagnosis of Nocardia based on the above criteria.

Other atypical pathogens, including actinomycetes, mycobacterium and fungus, should be considered in patients with chronic soft tissue infection and appropriate investigations include tissue biopsy for histological and microbiological examination. These investigations require access to microbiology laboratories with the ability to perform microscopy, culture and susceptibility testing.

Despite global improvements in laboratory capacity, many countries like TimorLeste will require significant assistance to strengthen their laboratories. The World Health Organization has identified laboratory strengthening as a priority. ${ }^{13}$

Although the burden of mycetoma in Timor-Leste is unknown, it is likely to be high as much of the population is impoverished, reside in a rural setting and have lim-
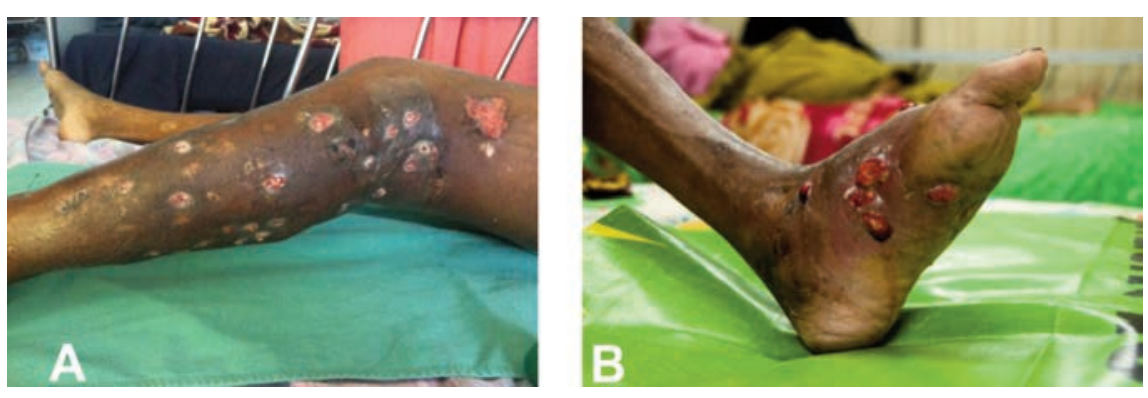

Figure 1. Extensive multiple draining sinuses of the lower limb typical of findings consistent with a diagnosis of Mycetoma. A) patient 2; B) patient 1.

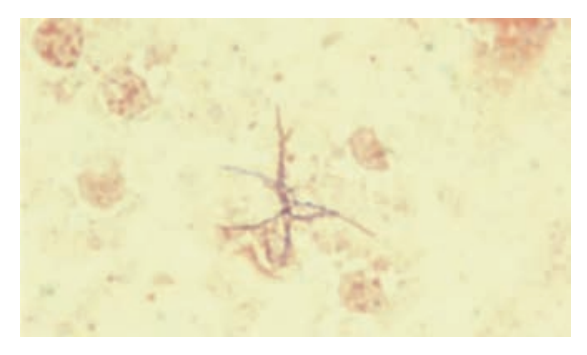

Figure 2. Branching beaded gram-positive filamentous bacteria consistent with the diagnosis of Nocardia spp. infection. 
ited access to health care. Strategies to prevent mycetoma in this region need to be further explored.

\section{Conclusions}

These cases highlight many features common to low to middle-income countries: Mycetoma is clinically underappreciated; it is a chronic illness that is associated with significant patient morbidity; diagnosis is difficult in regions of the world where laboratory capacity is limited and resource limitations compromise optimal medical management.

\section{References}

1. van de Sande WW, Fahal AH, Goodfellow $\mathrm{M}$, et al. The mycetoma knowledge gap: identification of research priorities. PLoS
Negl Trop Dis 2014;8:e2667.

2. Rattanavong S, Vongthongchit S, Bounphamala K, et al. Actinomycetoma in SE Asia: the first case from Laos and a review of the literature. BMC Infect Dis 2012; $12: 349$.

3. World Health Organization. Mycetoma Report by the Secretariat. Geneva: WHO; 2015.

4. Van de Sande WW. Global burden of human mycetoma: a systematic review and meta- analysis. PLoS Negl Trop Dis 2013;7:e2550.

5. Bonifaz A, Tirado-Sánchez A, Calderón L, et al. Mycetoma: experience of 482 cases in a single center in Mexico. PLoS Negl Trop Dis 2014;8:e3102.

6. Timor-Leste. Data [Internet]. Data.worldbank.org. 2018 [cited 1 October 2018]. Available from: http://data.worldbank.org/country/timorleste

7. World Health Organization. Global
Tuberculosis Report. Geneva: WHO; 2016.

8. Wilson J. Nocardiosis: Updates and Clinical Overview. Mayo Clin Proc 2012;87:403-7.

9. Baio P, Ramos JN, Santos LS, et al. Molecular Identification of Nocardia isolates from Clinical Samples and an Overview of human Nocardiosis in Brazil. PLoS Negl Trop Dis 2013;7:e2573

10. Palmer P, Reeder M. Imaging of tropical diseases. Heidelberg Germany: SpringerVerlag; 2001.

11. Sullivan DC, Chapman SW. Bacteria that masquerade as fungi: actinomycosis /nocardia. Proc Am Thorac Soc 2010;7:216-21.

12. Jorgensen J. Manual of clinical microbiology. Washington, DC: ASM Press; 2015.

13. World Health Organization. Global Action Plan on Anitmicrobial Resistance. Geneva: WHO; 2015. 